\title{
Digital demodulator unit of laser vibrometer standard for in situ measurement
}

\author{
Akihiro Oota ${ }^{1}$, Hideaki Nozato ${ }^{1}$, Wataru Kokuyama ${ }^{1}$, Yoshinori Kobayashi ${ }^{2}$, Osamu Takano ${ }^{2}$, Naoki \\ Kasai $^{2}$ \\ ${ }^{1}$ National Institute of Advanced Industrial Science and Technology, Central 3, 1-1-1 Umezono, Tsukuba, Japan \\ ${ }^{2}$ Neoark Corporation, 2062-21 Nakanomachi, Hachioji, Tokyo, Japan
}

ABSTRACT

To establish an easy-to-use traceability system for laser vibrometers in vibration acceleration standard, a highly accurate reference standard is required. In this study, we investigated the measurement reliability of a commercial laser vibrometer with an analog demodulator. The results confirmed a large measurement deviation from nominal sensitivity values due to characteristics of the analog demodulator. To reduce such deviation, a high-accuracy digital demodulator, which can be utilized as a reference standard, was developed. The experimental results confirmed the improved measurement accuracy of the developed digital demodulator.

\section{Section: RESEARCH PAPER}

Keywords: Laser vibrometer; analog demodulator; digital demodulator; ISO 16063-41

Citation: Akihiro Oota, Hideaki Nozato, Wataru Kokuyama, Yoshinori Kobayashi, Osamu Takano, Naoki Kasai, Digital demodulator unit of laser vibrometer standard for in situ measurement, Acta IMEKO, vol. 2, no. 2, article 11, December 2013, identifier: IMEKO-ACTA-02 (2013)-02-11

Editor: Paolo Carbone, University of Perugia

Received February $18^{\text {th }}, 2013$; In final form September $23^{\text {th }}, 2013$; Published December 2013

Copyright: ( 2013 IMEKO. This is an open-access article distributed under the terms of the Creative Commons Attribution 3.0 License, which permits unrestricted use, distribution, and reproduction in any medium, provided the original author and source are credited

Funding: This work was financially supported by METI (Ministry of Economy, Trade and Industry), Japan

Corresponding author: Akihiro Oota, e-mail: a-oota@aist.go.jp

\section{INTRODUCTION}

Laser vibrometers have been frequently used for precise vibration measurements to ensure the safety of commercial products in industry. The reliability and accuracy of such measurements should be guaranteed by a traceability system, which is a series of calibrations meeting national or international metrology standards regarding the use of laser vibrometers. To establish such a traceability system for laser vibrometers, a novel calibration method has been published as a new international standard (ISO 16063-41 [1]) for laser vibrometers. Some demonstrations have been attempted in accordance with this standard [2-5].

Laser vibrometers consist of two key components, a laser optics unit and a demodulator unit. A heterodyne laser interferometer or homodyne laser interferometer can be applied with a laser optics unit. The interferometry signal detected by a laser optics unit is transformed into an acceleration, velocity, or displacement signal by the demodulator unit. Two kinds of demodulator unit, analog and digital types, can be applied to realize this transformation.
For many years, many analog demodulator units have been used to transform interferometry signals into velocity signals. These units enable the direct conversion from Doppler signals to velocity signals, which can be continuously obtained over a wide velocity or frequency range. Such a laser vibrometer based on Doppler signals is referred to as a laser Doppler vibrometer. In an analog demodulator unit, the characteristics of the electric components such as the frequency-to-voltage converter, lowpass filter, and scaling amplifier strongly affect the measurement accuracy [6].

On the other hand, the digital demodulator unit applies a new signal processing method that does not depend on the characteristics of analog components. Because of the wide bandwidth of the interferometry signals, the only analog device required is an analog-to-digital converter (ADC) with high speed and high resolution. In the digital demodulator unit, the measurement accuracy depends mainly on the characteristics of the ADC and the calculation algorithm.

Therefore, the digital demodulator unit would enable users to easily achieve high measurement accuracy, in contrast with that obtained with the analog demodulator unit. However, 
digital demodulator units cannot always provide continuous measurements over the wide velocity or frequency range achieved by analog demodulator units because of technical barriers such as the limited number of samples available for digital processing and the limited sampling rate [6].

In this study, characteristics of various types of commercial laser vibrometer were investigated, and we clarify the disadvantages of the analog demodulator unit used. In addition, a novel digital demodulator unit, which enables us to continuously provide output data for in situ measurement, was developed to realize high measurement reliability, and we confirmed its capability experimentally.

\section{CHARACTERISTICS OF COMMERCIAL LASER VIBROMETER WITH ANALOG DEMODULATOR UNIT}

\subsection{Characteristics of three commercial laser vibrometers}

To confirm the potential of commercial laser vibrometers, we performed the calibration of a laser vibrometer and its demodulator unit. Three different kinds of commercial laser vibrometers with analog demodulators were applied to the calibration, which are in this work denominated LV A, LV B and LV $C$. These laser vibrometers are made by three manufacturers in Japan.

The primary calibration of the laser vibrometer using practical vibrations was performed in accordance with ISO 16063-41 (fringe counting method and sine approximation method). Figure 1 shows the experimental setup used for the primary calibration of the laser vibrometer. The laser interferometry system used as a reference standard is the homodyne laser interferometry system included in the Japanese national standard for primary calibration of accelerometers. In this setup, the fringe counting method was applied for the

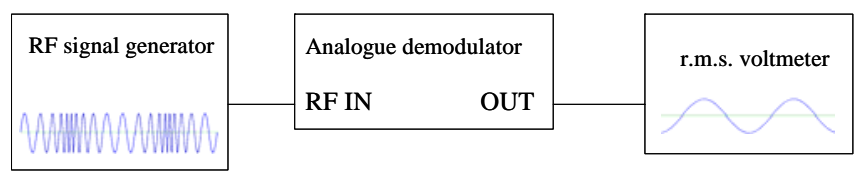

Figure 2. Experimental setup of demodulator unit calibration.

frequency range $20 \mathrm{~Hz}-80 \mathrm{~Hz}$, and the sine approximation method was applied for the frequency range $100 \mathrm{~Hz}-5 \mathrm{kHz}$.

In addition, their demodulator units are electrically calibrated with simulated frequency modulated signals, which are equivalent to output signals obtained from the laser optics unit in the calibration in accordance with ISO 16063-41. Figure 2 shows the experimental setup used for the demodulator unit calibration. A radio frequency (RF) signal generator was applied to generate the simulated frequency-modulated signal. An r.m.s. voltmeter (or digitizer) measures the output voltage of the demodulator unit. The RF signal generator and r.m.s. voltmeter can be calibrated through a traceability system of the time standard and voltage standard, respectively.

Figure 3 shows primary calibration results of the laser vibrometers LV-A, LV-B and LV-C, and also the electrical calibration results of their respective demodulator units. These demodulators were set for a nominal sensitivity of $10(\mathrm{~mm} / \mathrm{s}) / \mathrm{V}$. The calibration results of the demodulator units show very similar characteristics to the laser vibrometer calibration results.

Figure 4 shows the corrected primary calibration results based on the demodulator unit calibration results. Although the results from two different calibration methods had a large deviation of more than $1.0 \%$ from nominal sensitivities as shown in Figure 3, a small deviation of less than $0.5 \%$ was

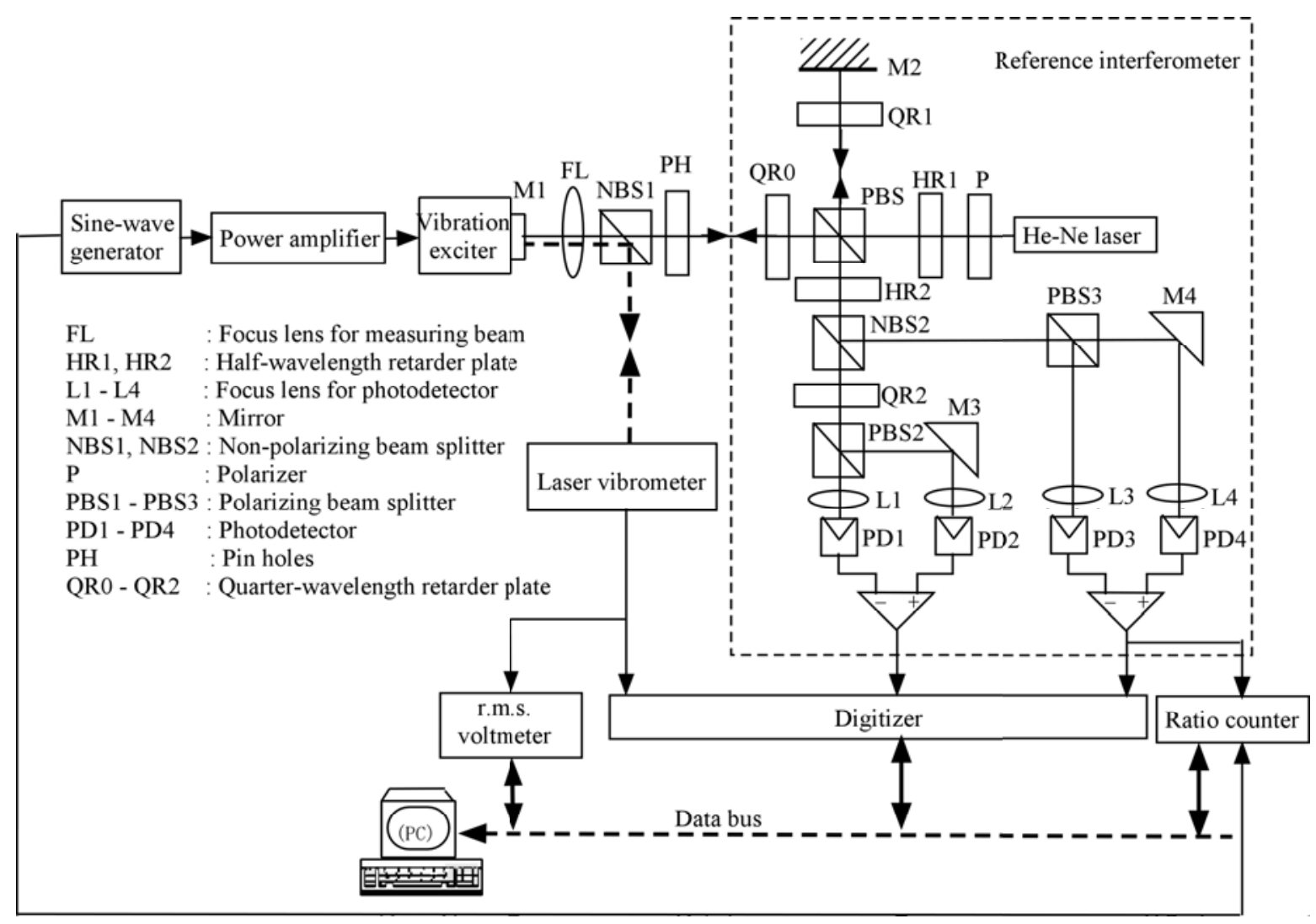

Figure 1. Experimental setup of primarv calibration of a laser vibrometer. 
almost achieved by performing a correction based on their demodulator unit calibration results.

The small deviation after correction in Figure 4 may normally be due to the effect of the optics unit of the laser vibrometer. However, all results after correction would be expected to be similar to each other. Therefore, the deviation after correction would be due more to the systematic error from common components in the demodulator unit calibration, such as the simulated frequency-modulated signal from the radio frequency signal generator, and not so much by the effect of the optics unit as by the systematic error from the common component in the demodulator unit calibration.

\subsection{Linearity and frequency dependency of the laser vibrometer}

The experiments mentioned above were carried out for a specific frequency series as accelerometer calibration. However, as the basic principle of a laser vibrometer, the frequency shift of light scattered from a moving surface by the Doppler-effect is proportional to the velocity of the moving surface. Therefore, the nonlinearity of the velocity amplitude may be more significant than the frequency characteristics. In this study, both typical characteristics were evaluated in detail for the LV-C. The setting of the nominal sensitivity value was fixed during the evaluation. Figure 5 shows the relative deviation from nominal sensitivity obtained while the velocity amplitude is adjusted from $3 \mathrm{~mm} / \mathrm{s}$ to $100 \mathrm{~mm} / \mathrm{s}$ at a calibration frequency of 160 $\mathrm{Hz}$. Nonlinearity higher than $5 \%$ was observed in this case.

Figure 6 shows the frequency characteristic of LV-C and its demodulator $C$ at constant velocity amplitude of $10 \mathrm{~mm} / \mathrm{s}$. The relative deviation from nominal sensitivity changes approximately $0.7 \%$ between $20 \mathrm{~Hz}$ and $5 \mathrm{kHz}$.

As the sensitivity fluctuation due to the velocity amplitude is much larger than that due to the frequency, the linearity for the velocity amplitude may be as important for in situ measurements as the sensitivity of the laser vibrometer. The evaluation results of the demodulator unit are very similar to the evaluation results of the laser vibrometer. As a result, we can conclude that the drastic fluctuation of the laser vibrometer sensitivity would be mainly due to the analog demodulator unit.

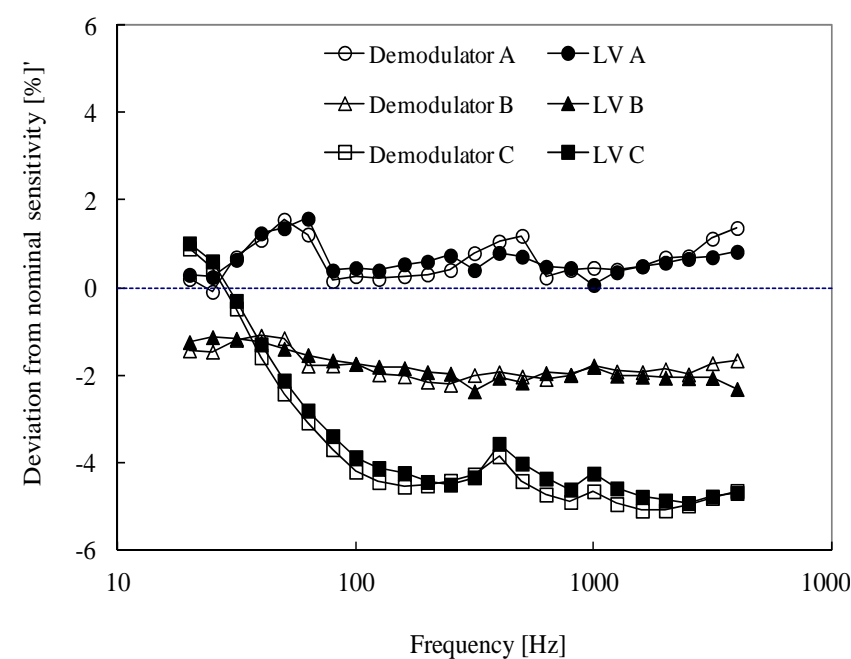

Figure 3. Comparison between calibrations of commercial laser vibrometers (LV) and electrical calibration of its demodulator unit calibration. The LVs of three different manufacturers were set at a nominal sensitivity of 10 $(\mathrm{mm} / \mathrm{s}) / \mathrm{V}$. A, B and C: different manufacturers. LV: Laser vibrometer.

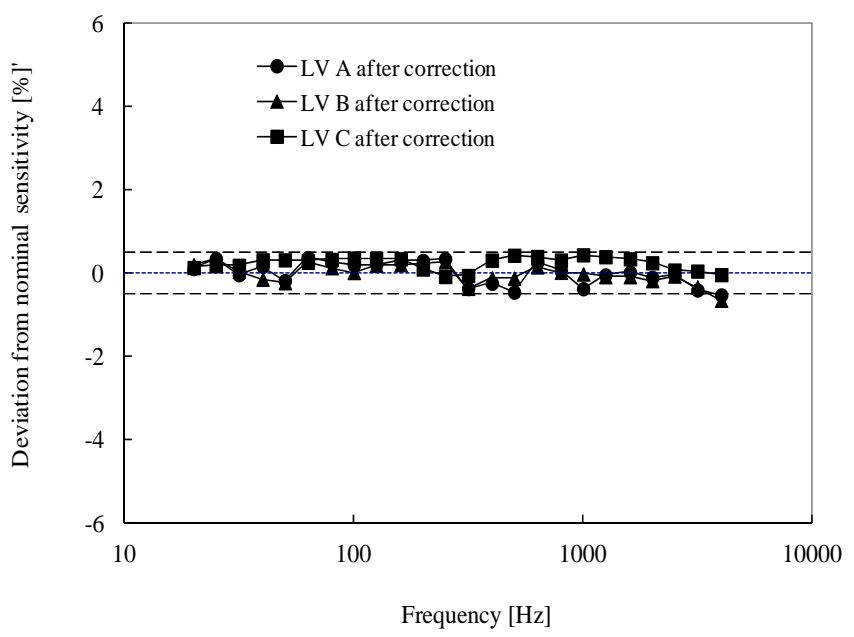

Figure 4. Correction results based on demodulator unit calibration.

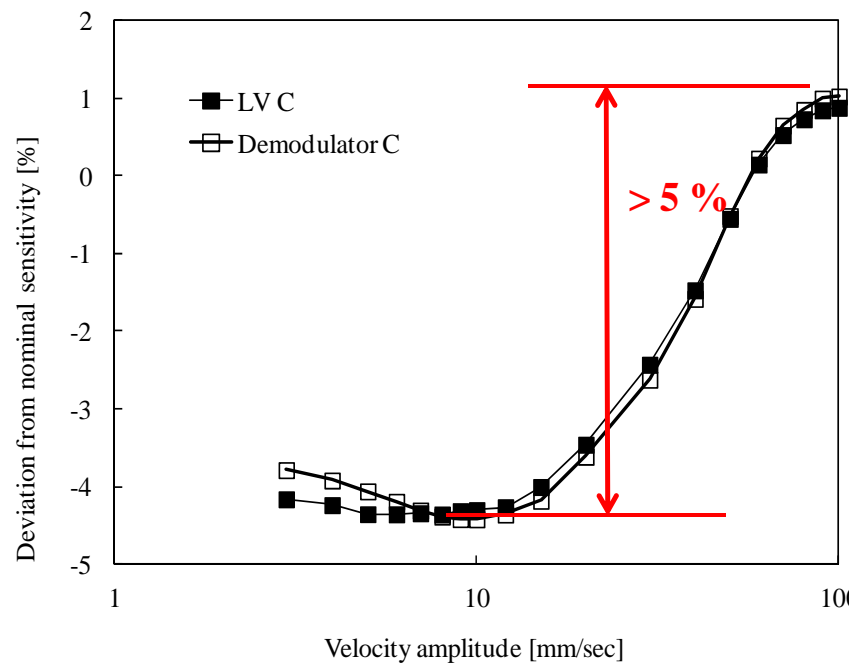

Figure 5. Typical nonlinearity for velocity amplitude at $160 \mathrm{~Hz}$ for a nominal sensitivity of $10(\mathrm{~mm} / \mathrm{s}) / \mathrm{V}$.

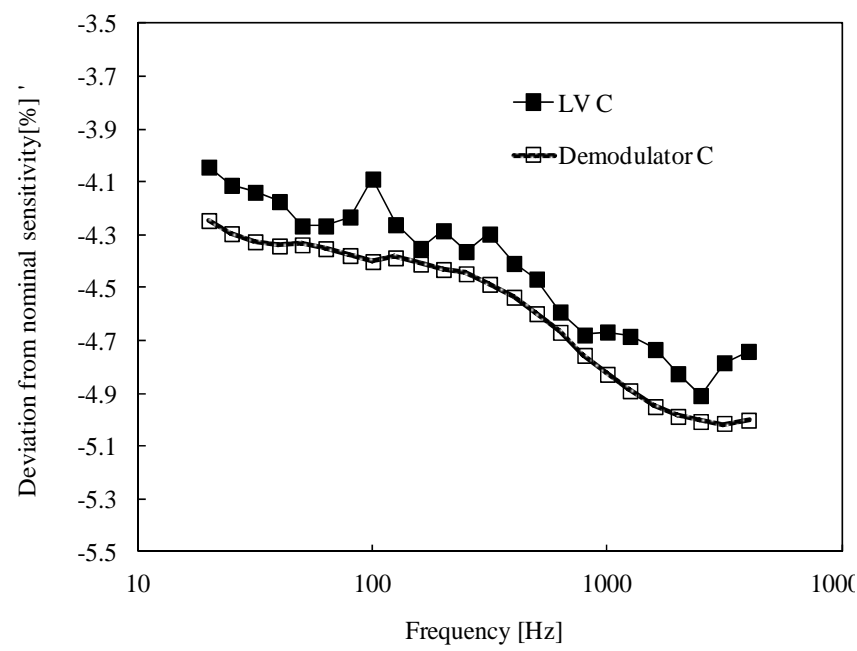

Figure 6. Typical frequency characteristic for nominal sensitivity of $10(\mathrm{~mm} / \mathrm{s}) / \mathrm{V}$ (Velocity amplitude of sinusoidal vibration given by vibration exciter is fixed at $10 \mathrm{~mm} / \mathrm{s}$ ). 
Consequently, to ensure the measurement reliability of the laser vibrometer in measurement ranges not covered by the primary calibration of the laser vibrometer in accordance with ISO 16063-41, the appropriate electrical calibration for analog demodulator units would be very useful to extrapolate the sensitivity. However, if a more precise vibration measurement is required, the measurement accuracy of the demodulator unit should be improved.

\section{DEVELOPMENT OF THE DIGITAL DEMODULATOR UNIT}

\subsection{Preliminary comparison between the digital demodulator unit and the analog demodulator unit}

In ISO 16063-41, the digital signal processing for the decoding of the Doppler signal is required in the definition of laser vibrometer standards. Therefore, to develop a higher accuracy demodulator unit, the potential of digital demodulation should be investigated by comparing it with analog demodulation. This comparison was carried out using electrical calibration and primary calibration explained above in Section 2 .

In this experiment, $\mathrm{LV}-\mathrm{C}$ was used as the laser vibrometer with the analog demodulator unit. The optics part of LV-C can be detached from the analog demodulator unit because it was specially designed for this purpose. On the other hand, to conveniently achieve digital demodulation, a commercial signal analyzer (Anritsu MS2690A) was applied. By using this instrument, I \& Q signals (in-phase and quadrature-phase signal pair) can be easily obtained, and the decoding of the I \& Q signals is then performed by our proprietary software using LabVIEW. The signal analyzer has an ADC resolution of 16 bits, a maximum sampling rate of $50 \mathrm{MHz}$, and an RF downconversion function. In this experiment, the signal analyzer and RF signal generator used to generate the simulated FM signal use a common sampling clock signal for their own processing.

To implement a laser vibrometer with a digital demodulator unit, the optics part of LV-C was connected to the signal analyzer.

Figure 7 shows the evaluation results obtained from both the primary calibration and electrical calibration. It compares the primary calibration results obtained for LV-C with the analog demodulator $\mathrm{C}$, with the analog demodulator $\mathrm{C}$ after

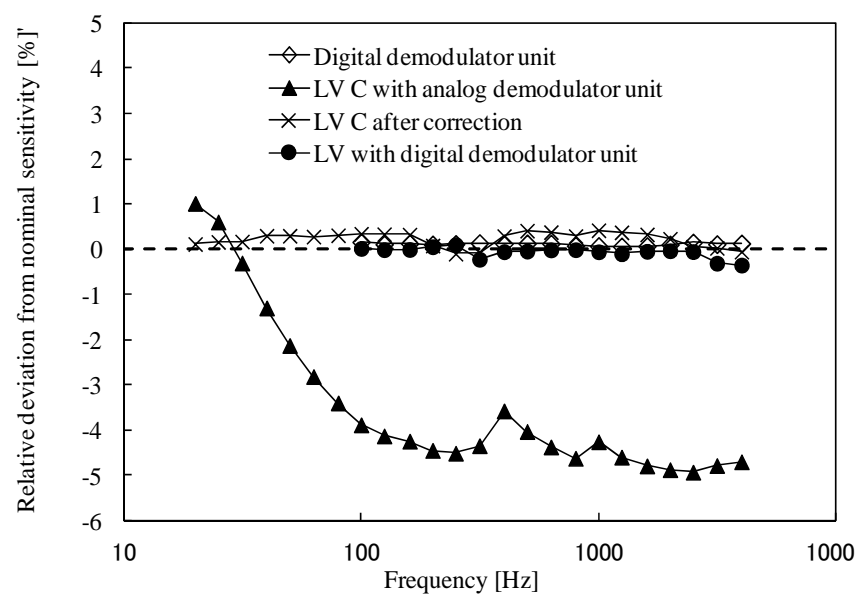

Figure 7. Preliminary comparison of digital demodulation with analog demodulation. correction, with the digital demodulator, and the electrical calibration result of only the digital demodulator unit. As a result, the potential of the digital demodulator unit to achieve higher measurement accuracy is confirmed.

\subsection{Development of the digital demodulator unit for in situ measurements}

Based on the results shown in Section 3.1, the digital demodulator unit with higher accuracy for laser vibrometer is now discussed. Although the application of the signal analyzer led to good results in the preliminary experiment, the signal analyzer would be unsuitable as it cannot continuously output data in real time, as required for in situ measurements. Therefore, a dedicated digital demodulator unit, which has a smaller deviation than commercial analog demodulator units, and which generates continuous output in real time, was designed for in situ measurements.

The developed digital demodulator unit is constructed with an ADC, digital to analog converter (DAC), and fieldprogrammable gate array (FPGA) at low cost. A He-Ne laser is used as the laser source in almost all commercial laser vibrometers in Japan. The driving frequency of its acousto optic modulator (Bragg cell) in the laser optics unit is fixed at 80 MHz. The maximum frequency shift due to the Doppler effect, which is equivalent to the maximum measurable velocity, is set to $10 \mathrm{MHz}$ to cover the velocity range below $3 \mathrm{~m} / \mathrm{s}$. Thus the maximum frequency of the signal to be detected by the ADC is equivalent to $90 \mathrm{MHz}$. To satisfy the Nyquist sampling theorem, a sampling rate of more than $200 \mathrm{MHz}$ is required as the maximum sampling rate of the ADC at least. To implement the measurement with higher accuracy in this study, the ADC is selected to have two channel inputs, a high sampling rate of 500 $\mathrm{MS} / \mathrm{s}$, and a high resolution of 12 bits. The DAC has a high sampling rate of $250 \mathrm{MS} / \mathrm{s}$ and a high resolution of 16 bits. High-speed signal processing is required to obtain the demodulation signal in as close to real time as possible. FPGA can process data faster than DSP. Therefore, an FPGA was applied to achieve high-speed signal processing for demodulation at low cost. The data processing including the digital demodulation algorithm embedded in the FPGA was developed to satisfy the requirement for the laser vibrometer standard described in ISO 16063-41. Additionally, ADC, DAC and FPGA are driven with common clock signal.

The specifications of the ADC, DAC and FPGA are given to enable continuous measurements over a wide frequency range of up to several hundred $\mathrm{kHz}$. This digital demodulator unit can receive a carrier frequency signal of either $80 \mathrm{MHz}$ or $10 \mathrm{MHz}$, which will enable it to immediately process the frequency signal after down conversion. In addition, the higher accuracy measurement can be achieved by applying a highaccuracy $10 \mathrm{MHz}$ external clock to this demodulator unit. Figure 8 shows a photograph of the developed digital demodulator unit.

The developed digital demodulator unit was evaluated using the same experimental setup in the previous section under the same experimental conditions as the analog demodulator unit. Figure 9 and Figure 10 show typical evaluation results obtained.

Experimental results showed that the relative deviation is confirmed to be below $0.3 \%$ for three frequency series in the applicable measurement range, and the time delay of the output due to the signal processing is evaluated to be about $30 \mu \mathrm{s}$. These results indicate that the developed digital demodulator 


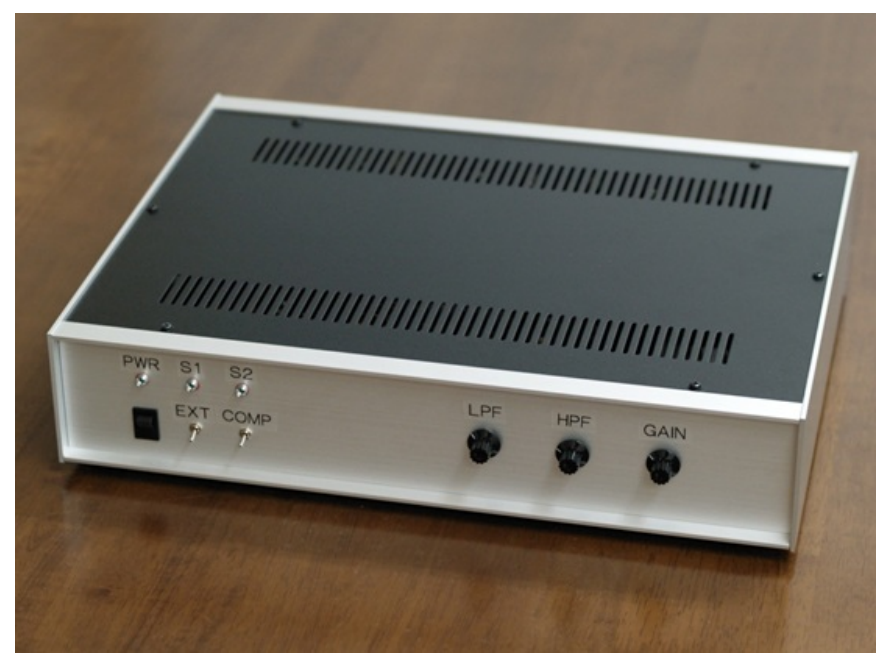

(a) Overview of the developed digital demodulator unit

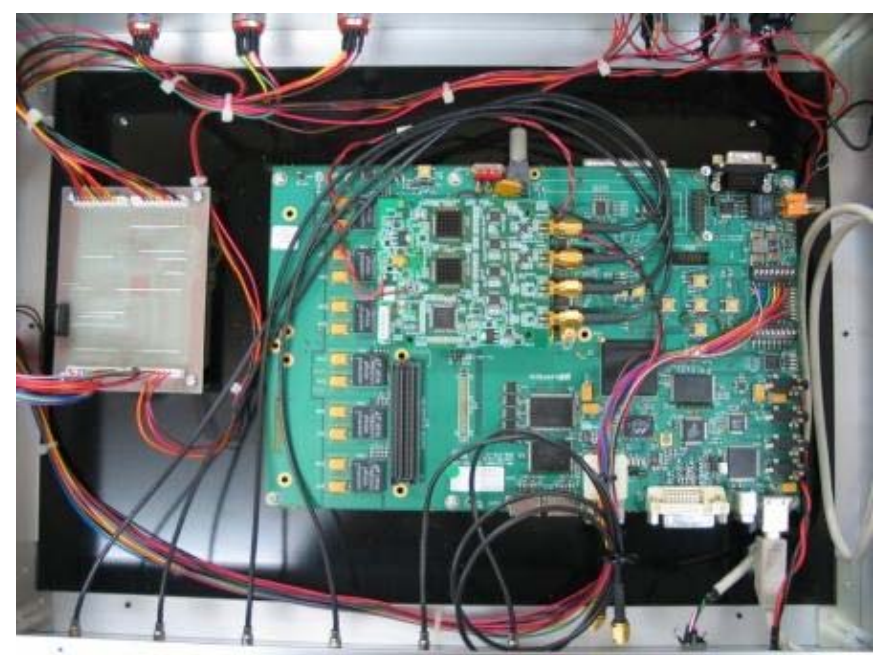

(b) Internal view of the developed digital demodulator unit

Figure 8. Digital demodulator unit for the laser vibrometer developed in this study.

unit has a sufficiently high measurement accuracy to be applicable as part of a laser vibrometer standard.

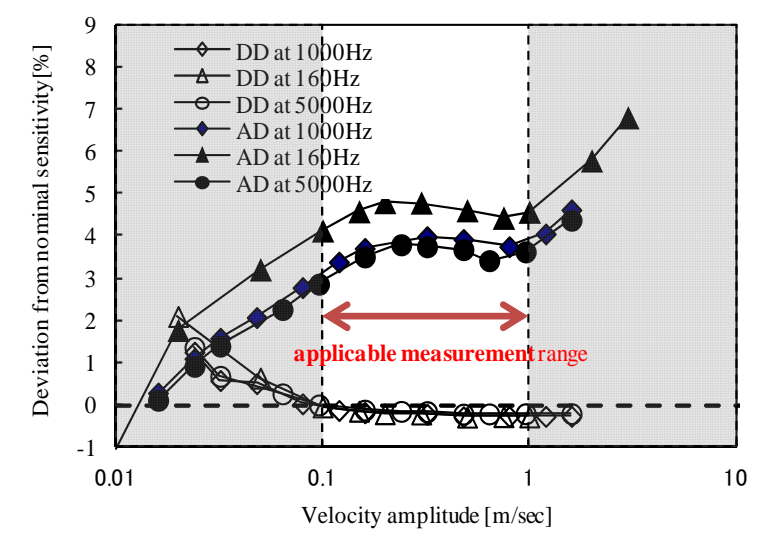

Figure 9. Typical evaluation results of the relative sensitivity deviation for the developed digital demodulator unit. DD: digital demodulator unit. AD: analog demodulator unit.

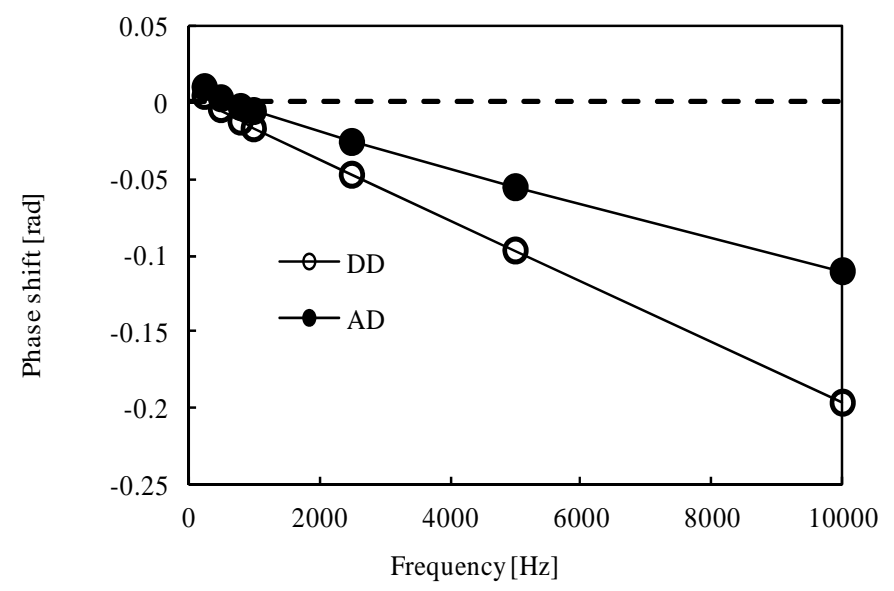

Figure 10. Typical evaluation results of the phase shift for the developed digital demodulator unit (phase shift). DD: digital demodulator unit. AD: analog demodulator unit.

\subsection{Practical shock measurement using a laser vibrometer with the developed digital demodulator unit}

The prototype of the developed digital demodulator unit must be combined with the laser optics unit to work as a laser vibrometer for in situ measurements. Therefore, the performance of the laser vibrometer, which consists of the developed digital demodulator unit and a commercial laser optics unit, was demonstrated using a shock acceleration exciter. To generate a rectilinear motion for sensing, a shock acceleration exciter was used for primary shock acceleration calibration as a national standard in Japan [7]. To validate the capability for sensing, in this experiment, a homodyne laser interferometer in a shock acceleration calibration system was used as the reference standard.

Figure 11 shows typical experimental results. The peak

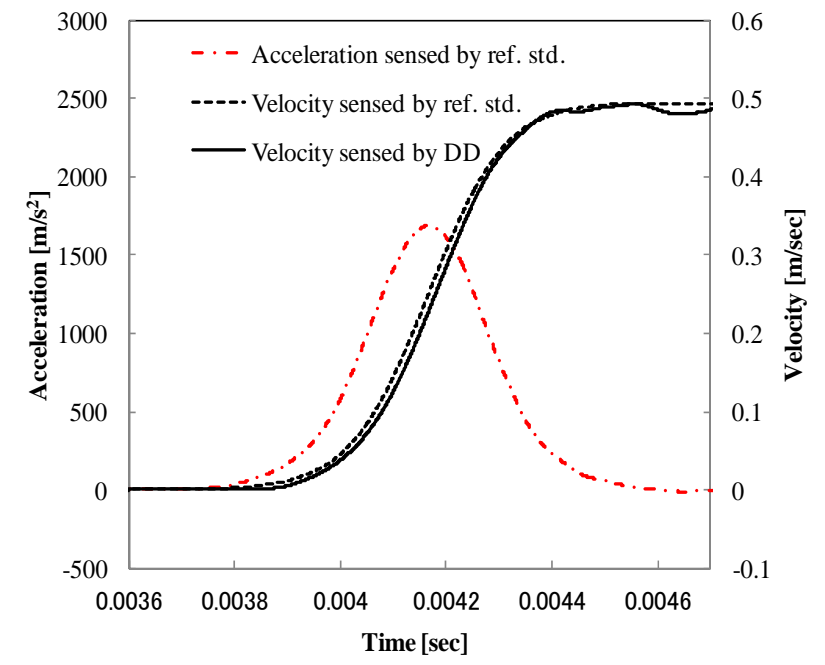

Figure 11. Comparison of typical measurement results of the laser vibrometer with prototype of the developed digital demodulator unit and shock acceleration standard with homodyne laser interferometer in NMIJ. DD: laser vibrometer with prototype of the developed digital demodulator unit. Ref.std.: homodyne laser interferometer for the shock acceleration standard in NMIJ. 
acceleration sensed by the reference standard was about $1700 \mathrm{~m} / \mathrm{s}^{2}$. From a comparison of both velocity waveforms, a slight time delay was observed in the laser vibrometer with the developed digital demodulator unit. This result is in agreement with the result of the phase shift obtained in Figure 10. In addition, the waveform that was obtained indicates unstable behavior after the application of shock acceleration. This instability may be due to the nonlinearity of the sensitivity to small velocity changes, and is a future technical issue to be investigated.

\section{CONCLUSIONS}

The characteristics of a laser vibrometer with an analog demodulator unit were investigated using three different commercial laser vibrometers. The calibration results of these demodulator units show very similar characteristics to those of the laser vibrometer calibration. Most of the deviation from the nominal sensitivity is due to the characteristics of the demodulator unit used.

The measurement accuracy of the laser vibrometer with a digital demodulator unit was compared with that of a laser vibrometer with an analog demodulator unit using the same laser optics unit. The results confirmed the higher measurement accuracy of the digital demodulator unit.

To overcome the difficulty of realizing a continuous signal processing for in situ measurement, the digital demodulator unit was developed using an FPGA, an ADC with a high sampling rate, and a DAC with a high sampling rate. The excellent potential of the developed digital demodulator unit was experimentally confirmed. On the other hand, we observed unstable behavior on the application of shock acceleration. This instability should be addressed to ensure greater usefulness for a laser vibrometer standard.

\section{ACKNOWLEDGEMENT}

This work was performed by AIST, and is a research project aimed at supporting small and medium companies. We acknowledge the financial support provided by the METI (Ministry of Economy, Trade and Industry). We deeply appreciate the kind cooperation of the two main Japanese manufacturers of laser vibrometers. We also wish to thank Dr. H-J.von Martens, who drafted ISO 16063-41, for the useful discussions.

\section{REFERENCES}

[1] ISO 16063-41: Methods for the calibration of vibration and shock transducers - Part 41: Calibration of laser vibrometers, International Organization for Standardization (2011).

[2] H.-J. von Martens, T. Bruns, A. Taubüner, W. Wabinski, U. Göbel, Recent progress in accurate vibration measurements by laser techniques, 7th International Conference on Vibration Measurements by Laser Techniques: Advances and Applications, Ancona, Italy, 2006, Vol. 6345, pp. 634501-1-23.

[3] A. Oota, T. Usuda, T. Ishigami, H. Nozato, H. Aoyama, and S. Sato, Preliminary implementation of primary calibration system for laser vibrometer, 7th International Conference on Vibration Measurements by Laser Techniques: Advances and Applications, Ancona, Italy, 2006, Vol. 6345, pp. 634503-1-8.

[4] U. Buehn, and H. Nicklich, "Calibration of laser vibrometer standards according to the standardization project ISO 1606341", 7th International Conference on Vibration Measurements by Laser Techniques: Advances and Applications, Ancona, Italy, 2006, Vol. 6345, pp.63451F-1-9.

[5] G. P. Ripper, G. A. Garcia, and R. S. Dias, The development of a new primary calibration system for laser vibrometer at INMETRO, IMEKO $20^{\text {th }}$ TC-3, $3^{\text {rd }}$ TC-16 and $1^{\text {st }}$ TC-22 International Conference, Mérida, Mexico, 2007, paper ID-105.

[6] M. Bauer, F. Ritter, and G. Siegmund, High-precision laser vibrometers based on digital Doppler-signal processing, $5^{\text {th }}$ International Conference on Vibration Measurements by Laser Techniques: Advances and Applications, 2002, Vol. 4827 pp. 50-61.

[7] H. Nozato, T. Usuda, A. Oota, T. Ishigami, Calibration of vibration pick-ups with laser interferometry Part IV: Development of shock acceleration exciter and calibration system, Measurement Science and Technology, Vol. 21, No. 6, (2010), paper ID 065107, pp. 1-10. 